\title{
Anomalous Decay Behavior of the Polypropylene Radicals Produced by Mechanical Fracture: Effect of the Excess Charges Due to Triboelectricity
}

\author{
Masato SaKaguchi and Junkichi Sohma \\ Faculty of Engineering, Hokkaido University, \\ Sapporo 060, Japan.
}

(Received December 23, 1974)

\begin{abstract}
Anomalous production of free radicals with increasing temperature in the range from $100^{\circ} \mathrm{K}$ to $173^{\circ} \mathrm{K}$ was observed in the decay process of the polypropylene mechano-radical produced by sawing in liquid nitrogen. It was experimentally demonstrated that this anomalous production of radicals at such low temperatures resulted from the excess electric charge due to the triboelectricity and from the oxygen dissolved in the liquid nitrogen. The reaction scheme for the radical production in this temperature range is presented as the formation of a peroxy radical from the carbanion produced by attachment of the excess electrons to the mechano-radical.
\end{abstract}

KEY WORDS Polymer Radicals / Polymer Reaction / ESR / Mechanical Fracture / Electric Charge / Friction /

In this decade it has been established ${ }^{1,2}$ that free radicals are produced by mechanical actions on solid polymers, such as large deformation, ${ }^{3,4}$ cutting, ${ }^{5,6}$ and ball-milling. ${ }^{7}$ A free radical produced by such mechanical actions is called a "Mechanoradical.' ESR provides a powerful experimental techique to study free radicals in polymers, ${ }^{8}$ and a lot of information on free radicals, e.g., identification of a radical species, a radical conversion, and decay of a radical, can be obtained from the analysis of an ESR spectrum. Species of the mechano-radicals of polyethylene (PE), polypropylene (PP), poly(tetrafluoroethylene) (PTFE), and poly(methyl methacrylate) (PMMA) were identified by analyses of the observed ESR spectra; all of the mechano-radicals from these polymers were found to be scission-type radicals, which were produced by ruptures of the main-chains. ${ }^{6,7}$ The creation of the scissiontype radicals from mechanical origins forms a clear contrast with production of the nonscission radicals, which are generally observed in the cases of ionizing radiation acting on the polymers. Relative intensities of ESR spectra observed at $77^{\circ} \mathrm{K}$ after heat treatments at various temperatures higher than $77^{\circ} \mathrm{K}$ are plotted against the temperatures of the heat treatments. The curve obtained by this procedure reflects well the decay behavior of the radical, because the ESR intensity is proportional to the concentration of radicals, which decay at higher temperatures due to unstability. This is the reason why this curve is called a "Decay Curve." Such a curve contains information on the characteristic decay hehavior of the radicals trapped in the polymer matrix, and the decay mechanism of these polymer radicals can be discussed from the analysis of the curve. ${ }^{9}$ Actually the decay curves of the radiation induced radicals of $\mathrm{PE},{ }^{9} \mathrm{PP},{ }^{10}$ and PTFE $^{11}$ demonstrate that the radicals decay with increasing temperature. Such decay with temperature is the normal behavior for the radicals, because the radicals are unstable intermediates which can survive only in either a trapped state or a frozen one. The decay curves were obtained for the radicals produced by the mechanical destruction of several polymers, such as PE, PP, and PTFE. It was found in these decay experiments of the mechano-radicals that they did not decay in a simple way but increased in concentration in the temperature range between $100^{\circ} \mathrm{K}$ and $173^{\circ} \mathrm{K}$. This means that some new 
radicals were produced by the heat treatment alone at temperatures in this range lower than room temperature. These anomalous increases in the decay processes are quite unique, these peculiar behaviors have been found not for the radicals produced by methods other than the mechanical fracture but do exist for all the mechano-radicals which we produce. ${ }^{6}$ Thus, one is led to believe that this anomalous decay behavior is one of the characteristics of the mechano-radicals. One can hardly imagine that a covalent bond can be broken by thermal motion at such a low temperature and a new radical could be thermally produced in the temperature range $77^{\circ} \mathrm{K}-173^{\circ} \mathrm{K}$. It is thus interesting to find a mechanism for this anomalous decay. Since the anomalous decay was most clearly observed for polypropylene, this polymer was chosen as an example. In oredr to elucidate the formation mechanism of new radicals at these low temperatures, some factors contributing to the anomalous behavior of the mechanoradical were looked for experimentally and successfully determined. The mechanism for this anomalous decay behavior will be discussed on the basis of the experimental results obtained.

\section{EXPERIMENTAL}

\section{Sample}

An isotactic polypropylene manufactured by Mitsui Chemicals was used. The antioxidantfree samples were prepared by extraction of the antioxidant with hot toluene.

\section{Apparatus}

Both 3BX and PE-3X ESR spectrometers made by JEOL were used with $100-\mathrm{KHz}$ modulation for ESR observation.

\section{Methods of Mechanical Destruction}

Sawing. Blocks of a polymer sample were sawed using a steel saw in a vessel filled with liquid nitrogen and the sawdust produced by this sawing was transferred along with some liquid nitrogen into an ESR sample tube, which had been kept in a Dewar containing liquid nitrogen. The sample tube containing the sawdust and liquid nitrogen was connected to a vacuum system and evacuated to $10^{-5}$ Torr. The sample tube was placed in the Dewar filled with liquid nitrogen during evacuation. By this procedure the sawdust of a polymer produced at $77^{\circ} \mathrm{K}$ and preserved at this temperature untill the warming experiments. The fractured sample obtained in this way were scarcely in contact with oxygen except for the impurities in the liquid nitrogen.

Ball-Milling. A special apparatus for ballmilling of a polymer was designed and built in our laboratory, in order to mill polymer flakes in a vacuum of $10^{-5}$ Torr at any temperature above $77^{\circ} \mathrm{K}$. The details of this apparatus have been described in the other paper. ${ }^{7}$

\section{Measurement of Electric Charge}

A vibrating-reed electrometer, TR-84M, and Faraday Cage, TR 8031, made by Takeda Riken Co. were used to measure the electric charge of the polymer sawdust.

\section{RESULTS AND DISCUSSIONS}

\section{Decay Curves}

Examples of decay curves of PP radicals are shown in Figure 1. In this diagram the ordinate represents the relative total intensity to the initial one at $77^{\circ} \mathrm{K}$ and the abscissa shows the temperatures of the heat-treatments, which was performed for $5 \mathrm{~min}$ at each temperature. ESR spectra were observed at $77^{\circ} \mathrm{K}$ after each heat treatment. The solid curve in Figure 1 is a decay curve of the PP mechano-radical produced by the sawing and the dotted line is that of the

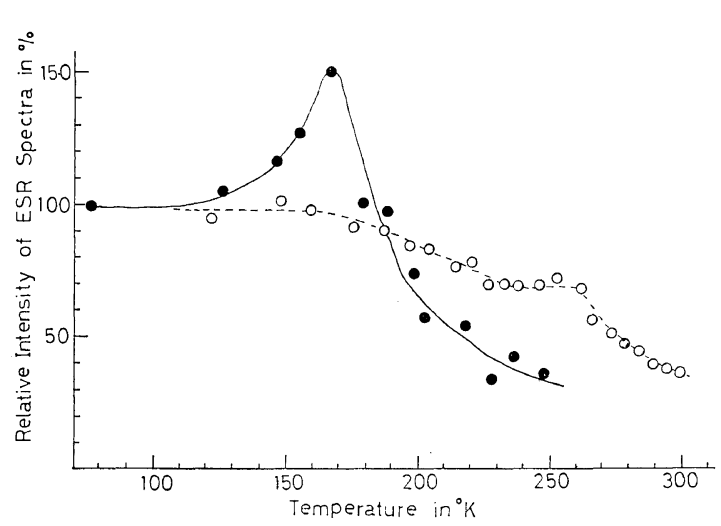

Figure 1. Decay curves of the PP radicals. The black circles correspond to the mechano-radical and the open circles to the radical produced by $\gamma$-rays. The abscissa is the temperature of the heat-treatment. 


\section{Sakaguchi and J. Sohma}

PP radical produced by $\gamma$-irradiation. The radicals produced by $\gamma$-irradiation decay step-wisely with increasing temperature of the heat-treatments; no increase of the ESR intensity is observed as a result of the heat treatments. This is commonly observed for other polymers and is a normal behavior in the decay of polymer radicals..$^{9,10}$ On the other hand, the decay curve of the mechano-radical increases to a maximum at $173^{\circ} \mathrm{K}$ and decreases more rapidly in the higher temperature region than that by $\gamma$-irradiation. This anomalous increase is a quite peculiar behavior in the decay process. It is very often observed that the ESR intensity is enhanced by a desaturation effect. Thus, careful observations were repeated with various $r-f$ powers and this anomalous increase was reproducibly observed even with the weakest power. This fact indicates that the increase in the ESR intensity originates not from the desaturation effect but from an increase of the radical concentration. It is worth mentioning that no such anomalous increases in the decay curve were observed for the radicals produced by $\gamma$-irradiation.

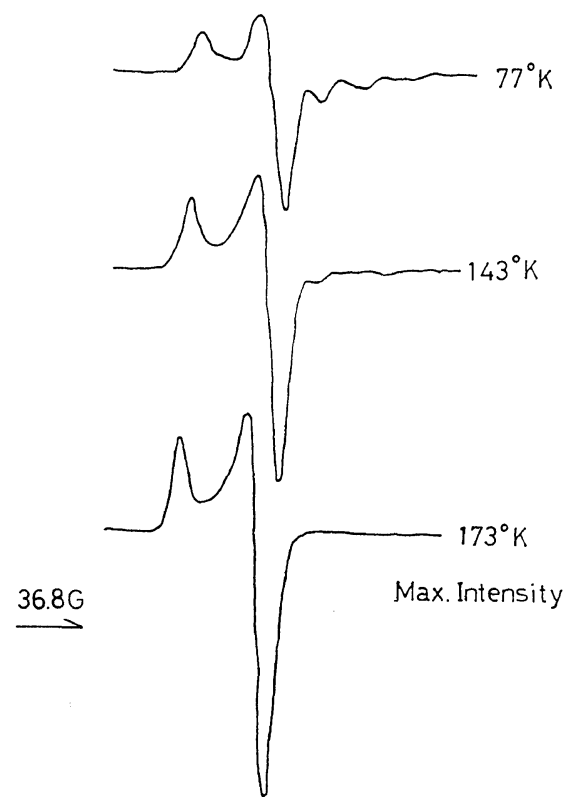

Figure 2. Changes in the line-shape of the ESR spectra after the heat-treatments below $173^{\circ} \mathrm{K}$. All observations were done at $77^{\circ} \mathrm{K}$. The temperatures shown in the figure are the heat-treatment temperatures.
It was also found that the ESR spectra changed in shape during this anomalous increase of the intensity. Examples of the line-shapes observed in this temperature region are shown in Figure 2. The temperatures shown in the figure are the heat-treatment temperatures and all the observations were made at $77^{\circ} \mathrm{K}$. The ESR spectrum observed before the heat treatment was decomposed into two components, which were identified to be an octet spectrum of the PP mechano-radical, $\underset{\mathrm{H}}{\mathrm{C}} \stackrel{\mathrm{H}}{\mathrm{C}} \mathrm{CH}_{3}$, and an asymmetric one from a peroxy radical..$^{6,12}$ During the heat treatment from $77^{\circ} \mathrm{K}$ to $173^{\circ} \mathrm{K}$ the spectrum of the PP mechano-radical is decaying and the asymmetric spectrum is increasing, as shown in Figure 2. At maximum intensity the observed spectrum became the single component from the peroxy radical. These experimetal results convince us that the peroxy radical is created by the heat-treatments even at such low temperatures (below $200^{\circ} \mathrm{K}$ ) after the sawing in liquid nitrogen.

Factors Affecting the Anomalous Behavior in the Decay Curve of the PP Mechano-Radical

Effect of Ingredients. Identical spectra and the same decay behavior were observed from the two samples, one of which the commercial sample without any extracting of the antioxidant and the other was the sample from which the antioxidant had heen removed. Thus, one can say that the antioxidants were not the source of the ESR spectra and had no effect on the anomalous behavior.

Electric Charge. Mechanical destruction is always accompanied by friction, which produces electric charge, especially on a polymer, by triboelectricity. Thus, it is quite probable that polymer samples after sawing are electrically charged. The electric charge of the sawdust of polypropylene was measured at $77^{\circ} \mathrm{K}$ by the electrometer with a Faraday Cage, and it was found that the polypropylene sample was electrically negative-charged after the sawing with the steel saw in liquid nitrogen. This excess charge produced on the sample at $77^{\circ} \mathrm{K}$ was gradually discharged at increased temperatures and became almost neutral near room temperature, as shown in Figure 3. In this figure the relative charge is plotted against the observation 


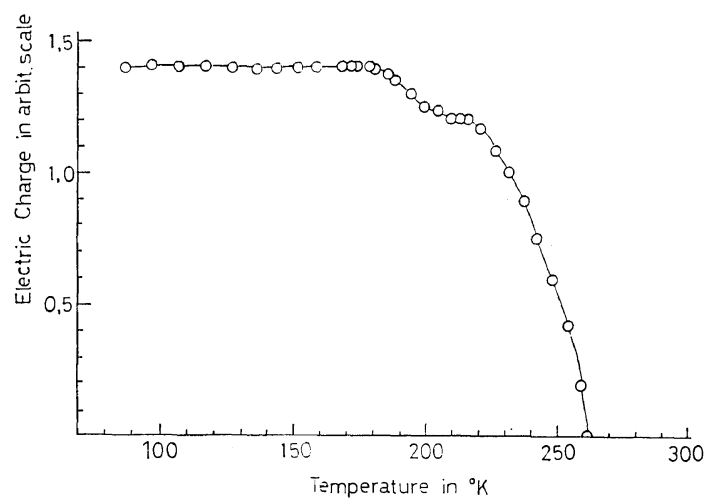

Figure 3. Discharge curve of the excess electriccharge due to the triboelectricity. The abscissa is temperature of observation and ordinate is an arbitrary scale.

temperatures, since the calibration of the Faraday Cage, which is valid only at room temperature, is not fully reliable at such low temperatures. It is interesting to notice that the electric discharge starts in the neighborhood of the temperature at which the radical decay starts. No characteristic ESR spectrum from trapped electrons was observed from the electrically charged sample, and the sample showed no blue color, which would be a characteristic absorption band of a trapped electron. It is concluded from these facts that the excess electrons, which arise from the negative charge of the sample, are not trapped and isolated in the polymer matrix but are associated with either molecules or radicals to form anions.

Effect of the Electric Charge on the Decay Curve

Decay curves were obtained for the same samples after fracture under the following two conditions:

(1) Insulation Experiment: The entire apparatus of sawing, including the experimenter, was electrically insulated from the ground. (2) Earth Experiment: The sawdust of the samples was not insulated but electrically grounded to the earth at $77^{\circ} \mathrm{K}$ before being transferred to an ESR sample tube. In the insulation experiment, of course, the excess charge survives more than in the earth experiment.

The two decay curves obtained from the experiments performed under the two different conditions are shown in Figure 4. The decay

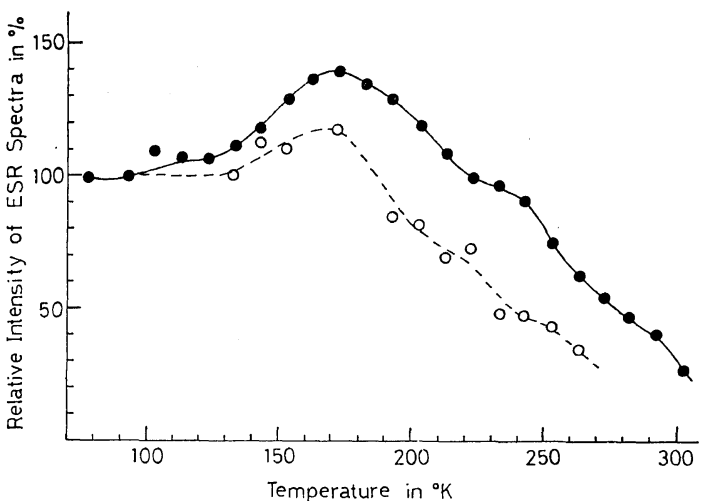

Figure 4. Decay curves obtained both in the insulation and the earth experiments. The abscissa is the temperature of the heat-treatment. The black circles correspond to the insulation experiment and the open circles to the earth experiment.

curve in the insulation experiment shows a more enhanced anomaly than that in the earth experiment. This comparison suggests that the production of the radicals by the heat-treatment is enhanced by the increased amount of the excess charge.

In order to confirm the effect of the excess charge on the anomalous phenomenon, an experiment on the effect of the electron scavenging was conducted. The polypropylene flakes were fractured in vacuum at $77^{\circ} \mathrm{K}$ with Tetracyanoethylene (TCNE), which is a strong electron scavenger, by the ball-milling method. The

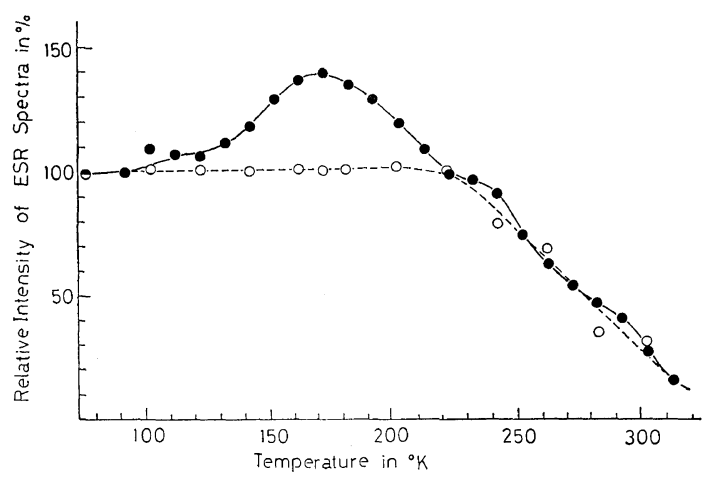

Figure 5. Comparison between the normal decay curve obtained by the ball-milling with TCNE the anomalous decay curve obtained without any scavenging of electrons. The lines connecting the black circles and the open ones are an anomalous decay and a normal one, respectively. 


\section{SAKaguchi and J. Sohma}

decay curve of the PP mechano-radical was obtained after the ball-milling with TCNE; the comparison between the decay curves with and without TCNE is made in Figure 5. In the case of milling with TCNE no anomalous increase was observed in the decay curve and the mechano-radicals stay unvaried up to about $250^{\circ} \mathrm{K}$ and then decayed. Apparently the normal decay was observed even for the PP mechano-radical, which had showed the anomalous behavior, when the excess electrons had been scavenged by the electron scavenger. The scavenging of
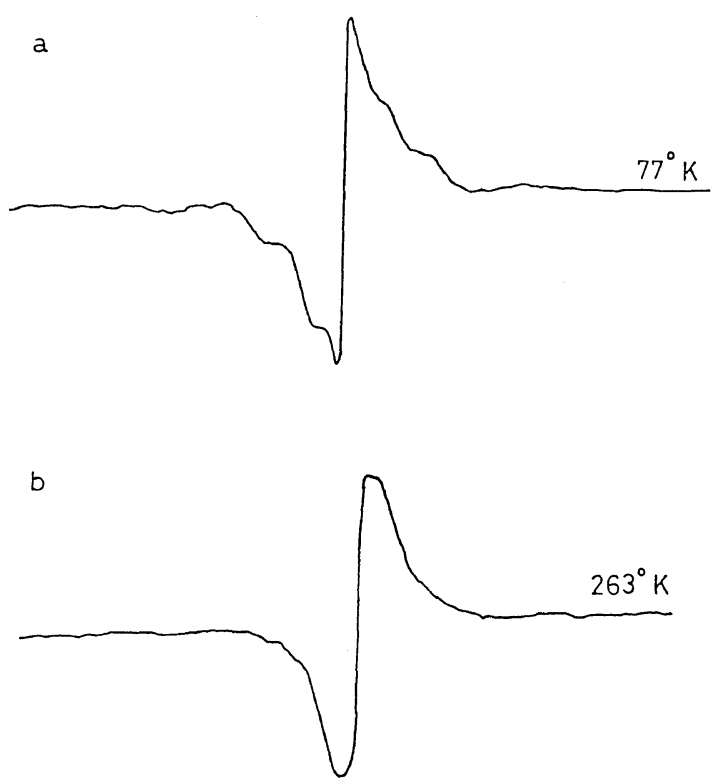

C

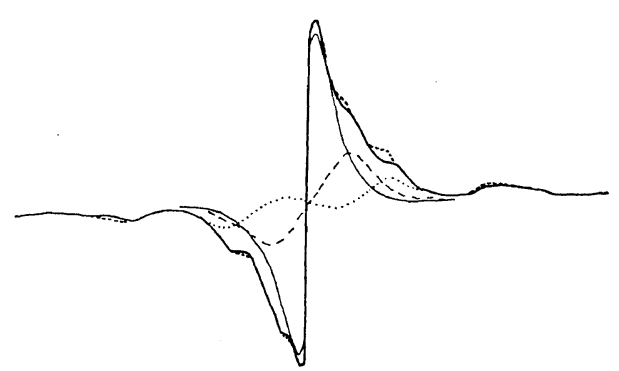

Figure 6. Observed ESR spectra after the ballmilling with TCNE and the decomposition of the spectrum: a, observed spectrum at $77^{\circ} \mathrm{K}$; b, observed spectrum after heat treatment at $263^{\circ} \mathrm{K}$; c, the decomposition of the spectra "a" into three components. the excess electrons by TCNE was verified experimentally by the analysis of the ESR spectra, shown in Figure 6. An ESR spectrum observed immediately after the ball-milling with TCNE at $77^{\circ} \mathrm{K}$ in vacuum is reproduced as Figure $6 \mathrm{a}$. After the heat treatment at room temperature the spectrum " $a$ " in Figure 6 was found to be converted into " $b$ " in the same figure. The ESR spectrum of " $b$ " is identical to the spectrum of a TCNE anion (TCNE) ${ }^{-}$, which can be produced by reduction of TCNE with sodium. On the basis of this change of the spectrum the ESR spectrum "a" was decomposed into the following three components: the sharp singlet from (TCNE) ${ }^{-}$, the octet from the PP mechanoradical, ${ }^{6}$ and a broad singlet from an unknown radical, shown as "c" in Figure 6. The evidence mentioned above indicates that the excess electric charge produced by the friction is at least one of the factors which contribute to the anomalous increase of the radicals by the heattreatment after the mechanical rupture.

Effect of Oxygen on the Decay Curve

The anomalous decay was most clearly observed in the case of sawing of the polypropylene blocks in liquid nitrogen, in which some oxygen might have become dissolved during the experiments. As shown in Figure 2, the new radical species which was created by the heat treatment was the peroxy-radical giving the asymmetric ESR spectrum. From this fact it is safe to

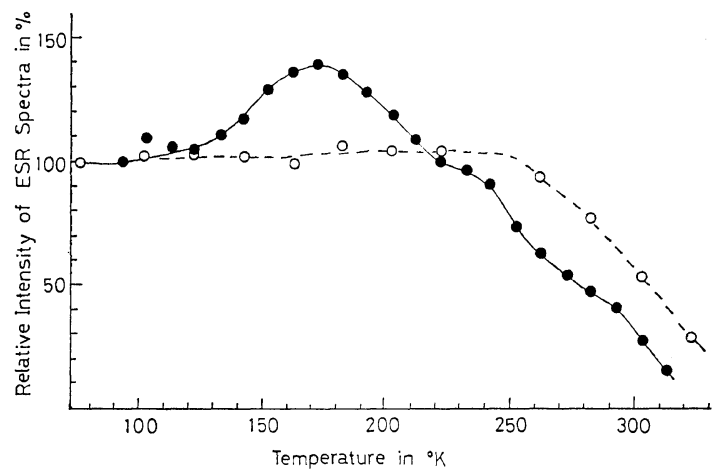

Figure 7. Comparison between the decay curve connecting the open circle obtained for the PP mechano-radical produced by the ball-milling at $10^{-5}$ Torr and the anomalous decay curve connecting the black circles, which is the same as the anomalous curve in Figure 5. 
assume that the oxygen molecules play an important role in this anomalous behavior in the decay process of the mechano-radical. In order to verify this assumption a comparison was made, as shown in Figure 7, between the decay curves obtained for the two different cases, namely fracture either in liquid nitrogen contaminated with oxygen or in vacuum of $10^{-4}$ Torr at $77^{\circ} \mathrm{K}$. The mechano-radical produced by the ball-milling at $77^{\circ} \mathrm{K}$ in vacuum does not show any anomalous increase but decays in the normal way. These facts demonstrate that the presence of oxygen molecules is necessary for the anomalous behavior in the decay of the PP mechanoradical. The experimental results mentioned in this section can be used to reach the conclusion that two factors, the excess electrons and the oxygen, are required for the observation of the anomalous increase in the decay curve of the mechano-radical.

\section{Mechanism for the Creation of Radicals Due to the Heat-Treatment}

As stated in the earlier part of this paper, the samples handled in the experiments were not electrically neutral but were negatively charged as a whole after the fracture. This was a big difference from ordinary samples, which are electrically neutral as a whole even if they contain free electrons, cations, or anions. And it was also found that the origin of the excess charge was not the trapped and isolated electrons. Thus, the negative charge must be in the form of anions. On the other hand, it has been experimentally demonstrated that mechanical. fracture of solid polypropylene at $77^{\circ} \mathrm{K}$ produces scissions of the main chains and creates a couple

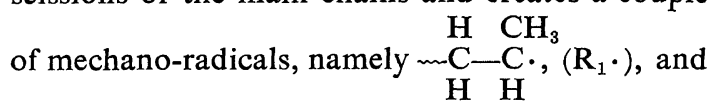
$\mathrm{H} \mathrm{CH}_{3}$ . rather large electron affinity, ${ }^{13}$ for example, $25 \mathrm{kcal} / \mathrm{mol}$ for a methyl radical. ${ }^{13,14}$ Therefore, it is most likely that the excess electrons are captured by the mechano-radicals to form anions, if there are excess charges. The arguments presented in the preceding paragraph convincingly suggest that the excess electrons produced by the triboelectricity during the mechanical destruction are combined with the mechano-radical and stabilized to form the carbanions, either $\mathrm{R}_{1}^{-}$or $\mathrm{R}_{2}^{-}$or both species, because of the electron affinity of such radicals. Although the exact values of the electron affinity of the mechano-radicals, $R_{1} \cdot$ and $R_{2} \cdot$, are not known, they are probably close to the value for the methyl radical. The carbanions, $\mathrm{R}_{1}{ }^{-}$and $\mathrm{R}_{2}{ }^{-}$, are not free-radicals and thus not observable by ESR. It is most reasonably considered that some of the mechano-radicals are converted into the carbanions immediately after the scission of the polymer chains by the combination with excess electrons and the ESR intensity observed in the initial state at $77^{\circ} \mathrm{K}$ is reduced by the number of the formed carbanions, $\mathrm{R}_{1}{ }^{-}$and $\mathrm{R}_{2}{ }^{-}$. It was also found, as mentioned in the last section, that the species of the radicals produced by the heat treatments was the peroxy radical. If the binding energies, $E_{\mathrm{B}}$, of the mechanoradicals, $R_{1} \cdot$ and $R_{2}$, with oxygen are larger than the electron affinities, $E_{\mathrm{A}}$, of these radicals, as shown in Figure 8, the electron captured by

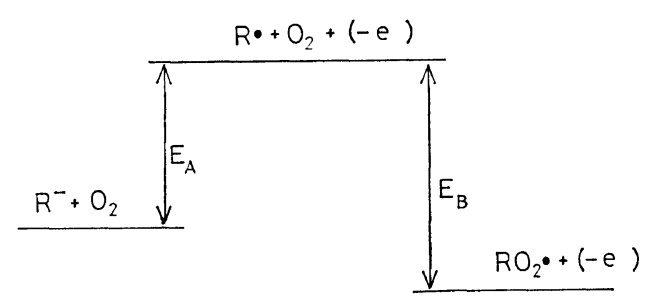

Figure 8. Assumed energy diagram for the system consisting of the radical, either $R_{1} \bullet$ or $R_{2} \bullet$, an oxygen molecule, and an excess electron. $E_{\mathrm{A}}$ and $E_{\mathrm{B}}$ are the electron affinity and the bond energy of the radical $R$, respectively.

the mechano-radicals can be released to form a peroxy radical and a free electron, if an activation energy is supplied from thermal energy at the elevated temperatures. In such a way the peroxy radicals are newly produced by the heat treatment, and the released electrons can easily leak to the ground at these temperatures. Through this process the ESR intensity is increased due to the creation of the peroxy radical from the non-radicals, the carbanions, only by the heat treatment. It has been found that the PP mechano-radicals, $R_{1} \cdot$ and $R_{2} \cdot$, readily react with oxygen molecules to produce the peroxy radical by heat treatment above $100^{\circ} \mathrm{K} .{ }^{12}$ Thus, 
both the original mechano-radical and the carbanions existing at $77^{\circ} \mathrm{K}$ are changed into the peroxy-radical by the heat treatment and the fraction from the carbanions is observed as the increase of the radicals by the heat treatment, which is the anomalous behavior in the decay process. It is worth noting that the oxygen plays two important roles in the anomalous decay: the first is the release of the excess electrons from the carbanion by the reaction with the radical and the second is the stabilization of the radical by forming the peroxy radical.

Excess charge due to the triboelectricity: $(-\mathrm{e})$

At the initial state of $77^{\circ} \mathrm{K}$

$\mathbf{R}_{1} \cdot$ the observed octet $^{12}$

$\mathrm{R}_{2} \mathrm{OO} \cdot$ : the asymmetric spectrum (only $\mathrm{R}_{2}$. reacts to $\mathrm{O}_{2}$ to form $\mathrm{R}_{2} \mathrm{OO} \cdot{ }^{12}$ )

$\left.\begin{array}{l}\mathbf{R}_{1} \cdot+(-\mathrm{e}) \longrightarrow \mathbf{R}_{1}^{-} \\ \mathbf{R}_{2} \cdot+(-\mathrm{e}) \longrightarrow \mathbf{R}_{2}^{-}\end{array}\right\}$no ESR spectra

Heat treatment in the temperature range of $100-173^{\circ} \mathrm{K}$

$$
\begin{aligned}
& \left.\begin{array}{l}
\mathrm{R}_{2} \mathrm{OO} \cdot: \text { unvaried } \\
\mathrm{R}_{1} \cdot+\mathrm{O}_{2} \longrightarrow \mathrm{R}_{1} \mathrm{OO} \cdot \text { : conversion to the peroxy radical }
\end{array}\right\} \text { ESR intesity unvaried } \\
& \left.\mathrm{R}_{1}{ }^{-}+\mathrm{O}_{2} \longrightarrow \mathrm{R}_{1} \mathrm{OO} \cdot+(-\mathrm{e})\right\} \text { creation of the peroxy radical from the nonradicals and } \\
& \left.\mathrm{R}_{2}{ }^{-}+\mathrm{O}_{2} \longrightarrow \mathrm{R}_{2} \mathrm{OO} \cdot+(-\mathrm{e})\right\} \text { increase of ESR intensity }
\end{aligned}
$$

Released electrons $(-\mathrm{e})$ leak to the ground and the sample approaches the neutral state

Heat treatment at temperatures above $173^{\circ} \mathrm{K}$

$\mathrm{R}_{1} \mathrm{OO}$. and $\mathrm{R}_{2} \mathrm{OO}$ : thermal decay

All excess electrons leak to the earth and the sample becomes electrically neutral

It should be pointed out that such anomalous decay was only observed for the mechano-radicals and no anomaly was observed for the radical produced by either $\gamma$-irradiation or $\mathrm{UV}$ illumination. This fact is also understandable if one remembers that the excess charge, from which the anomaly occurs, can only be produced by the triboelectricity accompanying the mechanical fractures. In the case of $\gamma$ and UV irradiation there is no excess change and the samples stay neutral even if ionization is generated in the samples. Therefore, no anomalous decay behavior was observed from the neutral samples.

\section{REFERENCES}

1. P. Y. Butyagin, A. A. Berlin, A. E. Kalmanson, and C. A. Blumenfeld, Vysokomol. Soedin, 1, 865 (1959).

2. H. H. Kausch, J. Macromol. Sci. Revs. Macromol. Chem., C4 (2), 243 (1970); a list of refernces will be found in this review paper.

3. A. Peterlin, Polym. Sci., C, No. 32, 297 (1970).

4. K. L. DeVries, ibid., No. 32, 325 (1970).

5. T. Kawashima, M. Nakamura, S. Shimada, H. Kashiwabara, and J. Sohma, Rep. Progr. Polym. Phys. Japan, 12, 469 (1969).

6. T. Kawashima, S. Shimada, H. Kashiwabara, anb J. Sohma, Polymer J., 5, 135 (1973).

7. M. Sakaguchi and J. Sohma, J. Polym. Sci., Part $A-2$, in press.

8. For example, "ESR Application to Polymer Research" (Noble Symposium 22) edited by Per-Olof Kinell, B. Rånby, Runnström-Reio, Almgvist Wiksell, Stockholm, 1972.

9. S. Nara, H. Kashiwabara, and J. Sohma, J. 
Polym. Sci., Part A-2, 5, 929 (1967).

10. S. Shimada, H. Kashiwabara, and J. Sohma, ibid., Part A-2, 3, 1291 (1970).

11. Unpublished data, T. Kawashima, Master's Thesis, College of Eng., Hokkaido Univ., 1968.
12. M. Sakaguchi, H. Yamakawa, and J. Sohma, Polym. Letters, 12, 193 (1974).

13. H. O. Pritchard, Chem. Rev., 52, 529 (1953).

14. N. S. Hush and J. A. Pople, Trans. Faraday Soc., 51, 600 (1955). 